\title{
Monolayer Excitonic Emission for Imaging Spatial Dispersion of Photonic Crystals
}

\section{Supporting Information}

\author{
Wenzhuo Huang, ${ }^{\dagger}$ Chawina De-Eknamkul, ${ }^{\ddagger}$ Xingwang Zhang, ${ }^{\ddagger}$ Eric \\ Leewong, ${ }^{\dagger}$ Meng-Qiang Zhao, " A. T. Charlie Johnson," and Ertugrul \\ Cubukcu, ${ }^{*},+\neq$ \\ $\uparrow$ Department of Electrical and Computer Engineering, University of California, San \\ Diego, La Jolla, California 92093-0407, United States \\ $\ddagger$ Department of NanoEngineering, University of California, San Diego, La Jolla, \\ California 92093-0448, United States \\ IDepartment of Physics and Astronomy, University of Pennsylvania, Philadelphia, \\ Pennsylvania 19104, United States \\ E-mail: ecubukcu@ucsd.edu
}

\section{Contents}

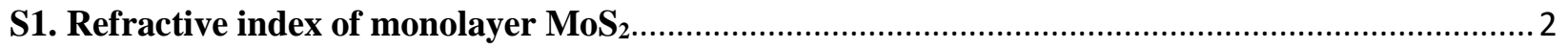

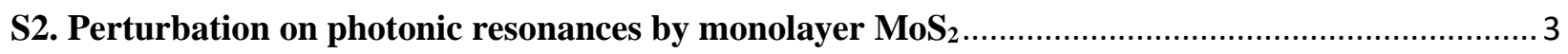

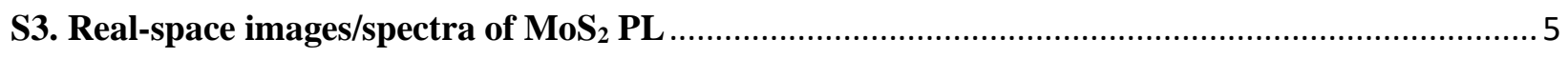

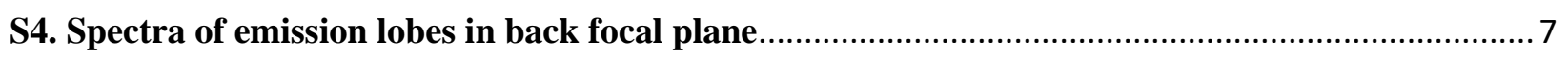

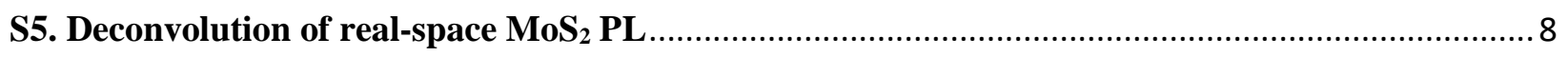

S6. Angle-resolved transmittance along $\Gamma$-M direction ............................................................... 9

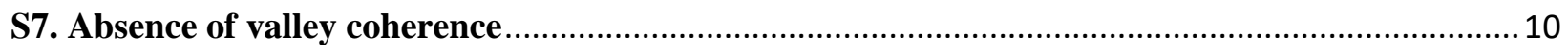

S8. $\mathrm{MoS}_{2}$ PL coupled with another TE-like band ...................................................................... 11

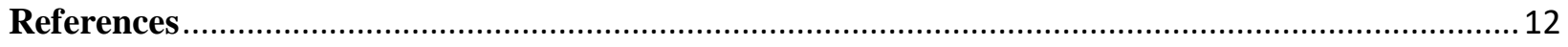




\section{S1. Refractive index of monolayer $\mathrm{MoS}_{2}$}

The dielectric constant $\varepsilon_{r}(E)=\varepsilon_{1}(E)+i \varepsilon_{2}(E)$ of monolayer $\mathrm{MoS}_{2}$ is extracted from reflection measurement by Lorentzian oscillators model

$$
\varepsilon_{r}(E)=\varepsilon_{\text {offset }}-\sum_{j=1}^{N} \frac{f_{j}}{E^{2}-E_{0 j}^{2}+i E \Gamma_{j}}
$$

where $\varepsilon_{\text {offset }}$ is the permittivity offset; $f_{j}, E_{0 j}$ and $\Gamma_{j}$ are the strength, energy and damping constant of $i^{\text {th }}$ oscillator.

The refractive index $\tilde{n}=n+i \kappa=\sqrt{\varepsilon_{r}}$ of $\mathrm{MoS}_{2}$ is plotted in Fig. S1.

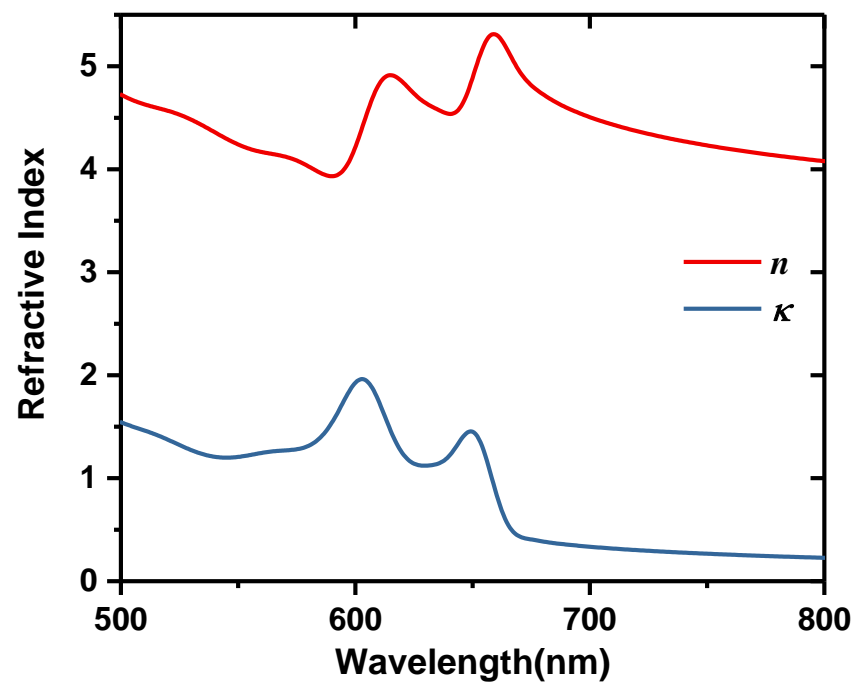

Fig. S1 Real and imaginary parts of the refractive index of monolayer $\mathrm{MoS}_{2}$. 


\section{S2. Perturbation on photonic resonances by}

\section{monolayer $\mathrm{MoS}_{2}$}

For better comparison between the pristine $\mathrm{PhC}$ and the one covered with monolayer $\mathrm{MoS}_{2}$, we choose the device with large enough area for both conditions. The microscopic image of the device is shown in Fig. S2a. The periodicity of is 520 $\mathrm{nm}$ and radius of the air holes is $120 \mathrm{~nm}$. The area within the orange square is the $\mathrm{PhC}$ and the blue dashed lines are the boundary of the $\mathrm{MoS}_{2}$ monolayer. About one third of the PhC slab is covered by $\mathrm{MoS}_{2}$.

At normal incident angle, the redshift of the resonance is $3.6 \mathrm{~nm}$. We could also observe a broader linewidth and a shallower dip of the resonance with monolayer $\mathrm{MoS}_{2}$. At $5^{\circ}$ to the normal direction, three modes with different depths and linewidths are observed. The redshifts are $5.3 \mathrm{~nm}, 3.6 \mathrm{~nm}$ and $5.8 \mathrm{~nm}$ respectively from left to right. The gaps between two transmission lines at $\sim 600 \mathrm{~nm}$ in Fig. S2b and at $\sim 650 \mathrm{~nm}$ in Fig. S2c are due to the absorption peaks in the imaginary part of refractive index (see $\kappa$ in Fig. S1). 

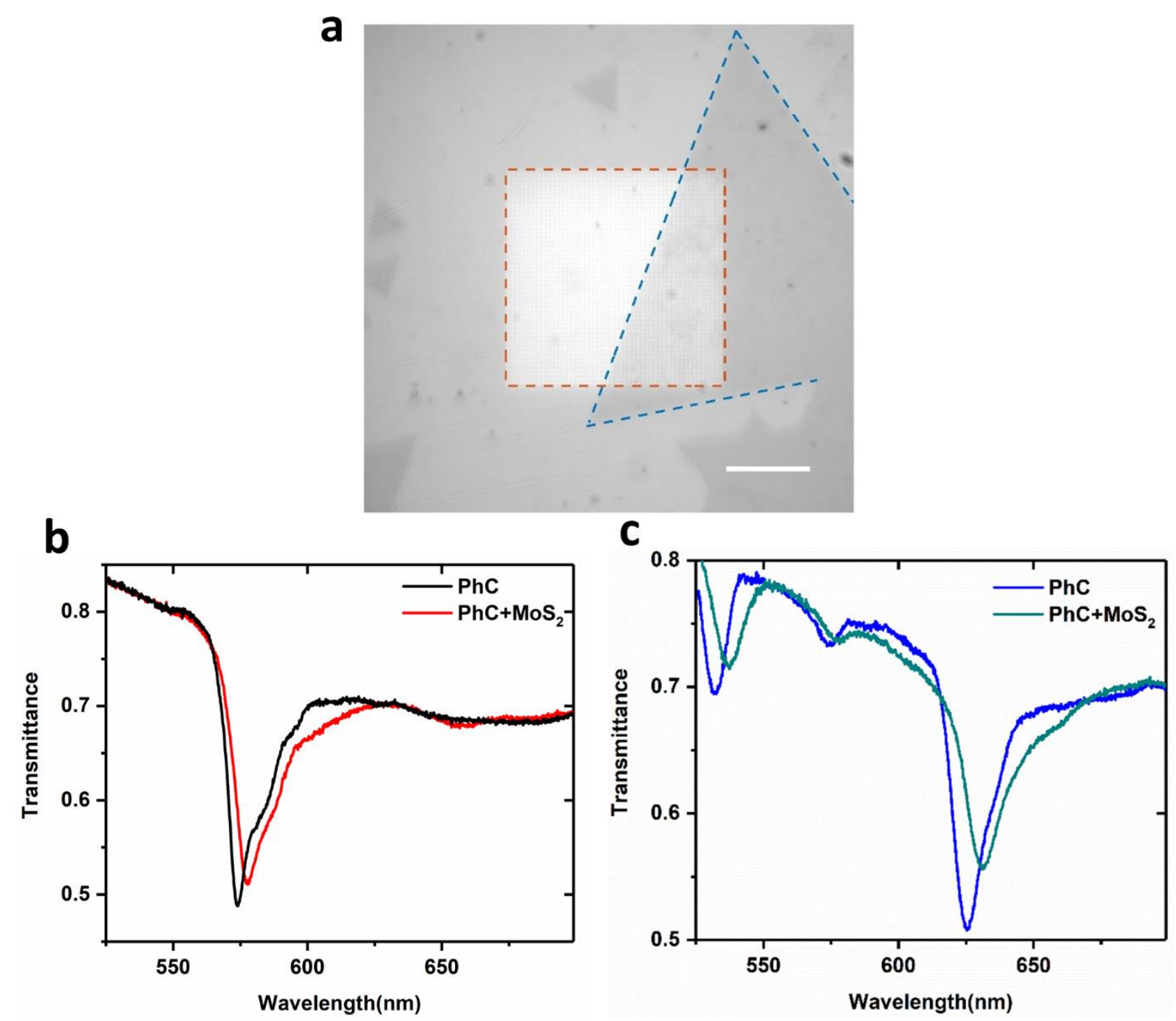

Fig. S2 (a) The microscopic image of the PhC sample partially covered with monolayer $\mathrm{MoS}_{2}$. The scale bar is $10 \mu \mathrm{m}$. (b) The transmission spectra at normal direction. (c) The transmission spectra at $5^{\circ}$ to the normal direction. 


\section{S3. Real-space images/spectra of $\mathrm{MoS}_{2} \mathrm{PL}$}

For real-space imaging and spectroscopy, we use another lens between the back focal plane and the entrance slit of the spectrometer. The focal length of this lens is half of the distance between the back focal plane and the entrance slit, which creates a part of a $4 \mathrm{f}$ system (the other part is not shown in the Fig. S3c for simplicity). Photons generated from same position in the sample plane are imaged onto a same spot on the CCD camera.

Figs. S3a and S3b show the real-space images of PL on and off the PhC. Because excitonic photons are coupled into the guided mode resonances of the $\mathrm{PhC}$, they can travel a sizable distance in the PhC slab and then leak into the free space. Thus, we see a wider spatial distribution of PL on the PhC. The PL distribution along the blue cutting lines in these two figures are shown in Fig. S3d, which has the same $\mathrm{x}$-coordinate as Figs. S3a and S3b. This PL distribution on the PhC will broaden the real-space PL spectra (Fig. S3e) as we increase the aperture width in order to capture the entire $\mathrm{PhC}$ region that contributes to the PL emission as shown in Fig. S3b. 

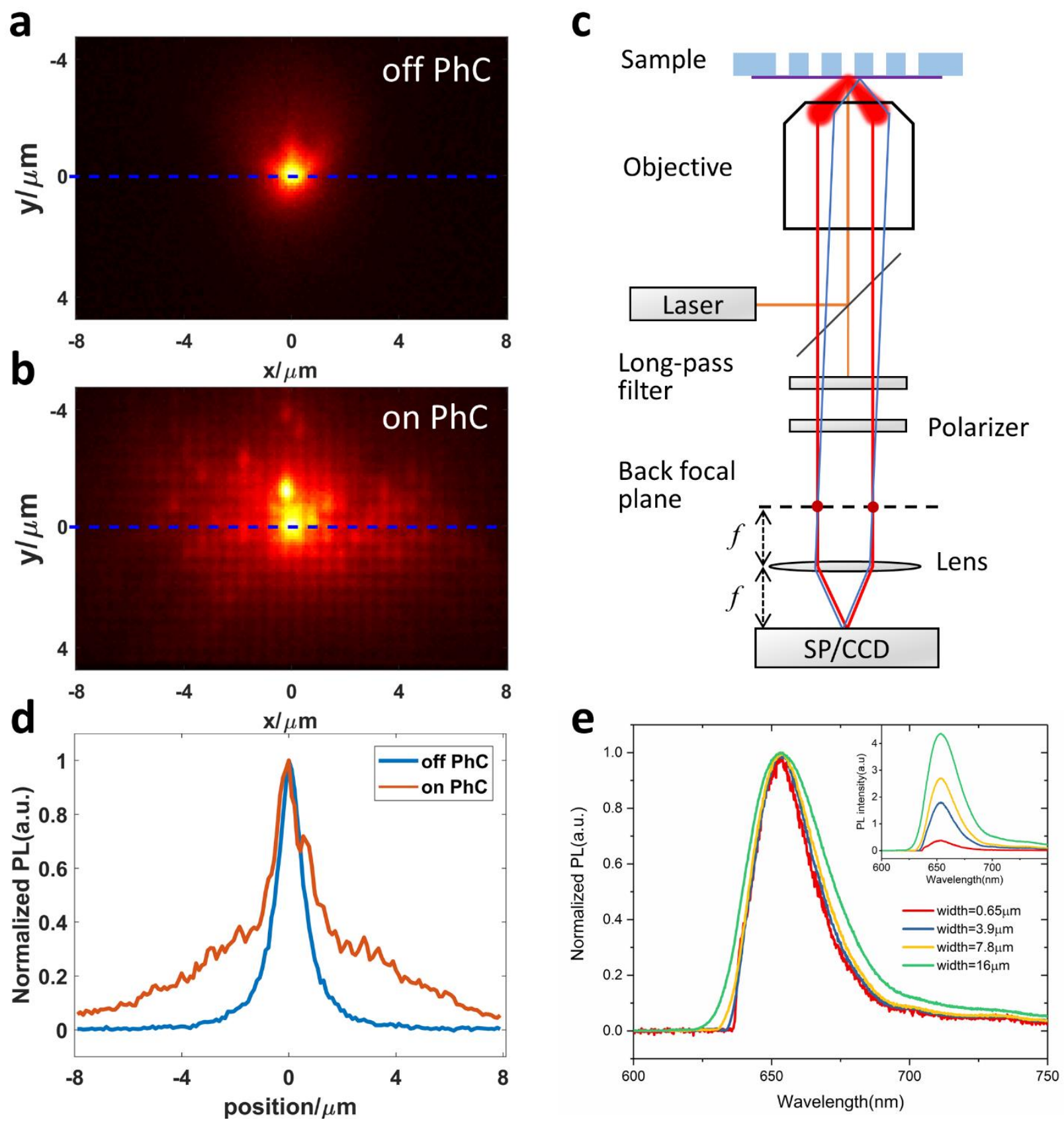

Fig. S3 Real-space image and setup. PL images (a) on the $\mathrm{PhC}$ and (b) off the PhC. (c) Experimental setup for real-space measurement. (d) Normalized PL intensity along blue cutting lines in (a) and (b). (e) Real-space PL spectra broadened with increasing aperture width. 


\section{S4. Spectra of emission lobes in back focal plane}

At the entrance slit of the spectrometer, we use a 2D aperture to select the area that we are interested in either the real or Fourier space depending on the configuration. PL spectra shown in Fig. 2a of the main text are collected using the aperture settings denoted as "aperture 1", which is centered at $k_{\mathrm{x}}=0$, in Fig. S5a (black rectangle marked as 1). For this arrangement, at each wavelength, photons with all perpendicular momentum components $k_{\mathrm{y}}$ are averaged out. Thus, the PL spectrum on the PhC in Fig. 2a can be deemed as the integral over all $k_{\mathrm{y}}$ (or angles) of Fig. $2 c$ of the main text.

Fig. S5b shows the normalized PL from emission lobes in $\Gamma$-X (aperture 2) and $\Gamma-\mathrm{M}$ (aperture 3) directions. Different peak positions can be seen in the spectra, showing excitonic photons coupled into different resonances (especially at bandedges).

a

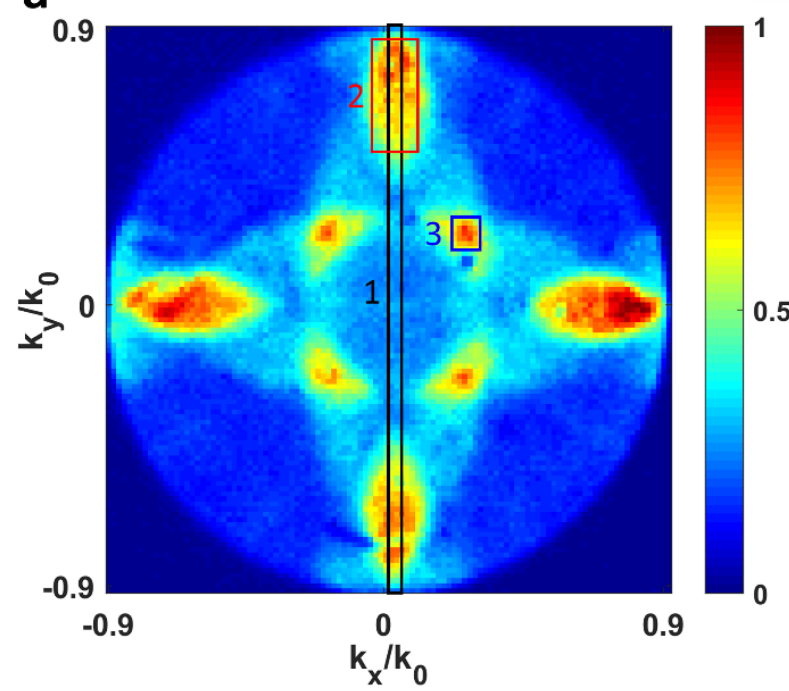

b

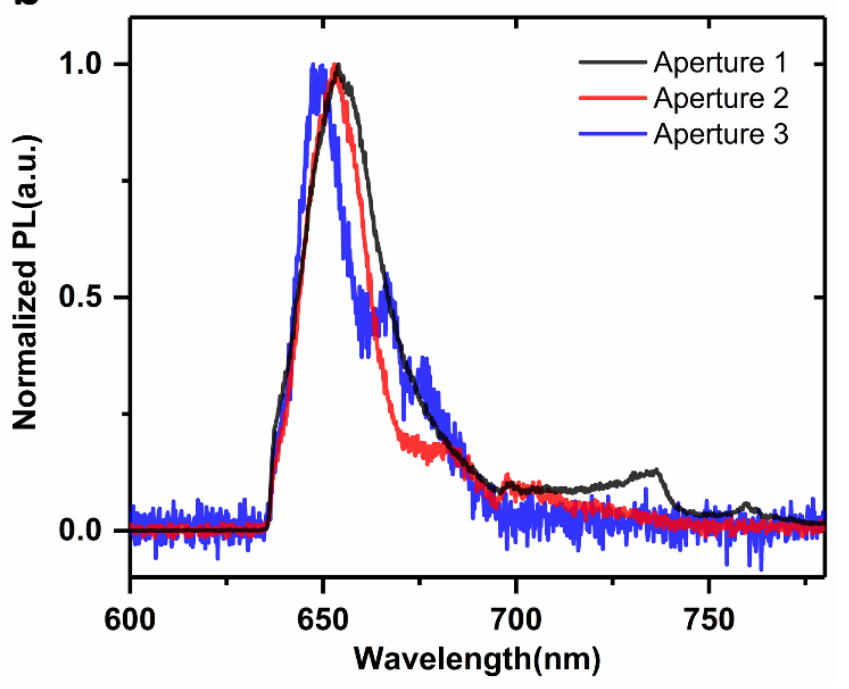

Fig. S5 (a) 2D Fourier plane corresponding to the excitonic PL emission. Different regions indicated by black (1), red (2), and blue (3) rectangles in 2D Fourier plane correspond to the aperture sizes and positions used for the PL spectra in (b). (b) Normalized PL spectra from each aperture. PL from aperture 1 is the same as Fig. 2a in the main text. 


\section{S5. Deconvolution of real-space $\mathrm{MoS}_{2} \mathrm{PL}$}

The real space PL spectrum of $\mathrm{MoS}_{2}$ on the $\mathrm{PhC}$ is deconvolved into two Lorentzian peaks. The central wavelength of the A exciton peak is $652 \mathrm{~nm}$ and that of the $\mathrm{A}^{-}$trion is $665 \mathrm{~nm}$.

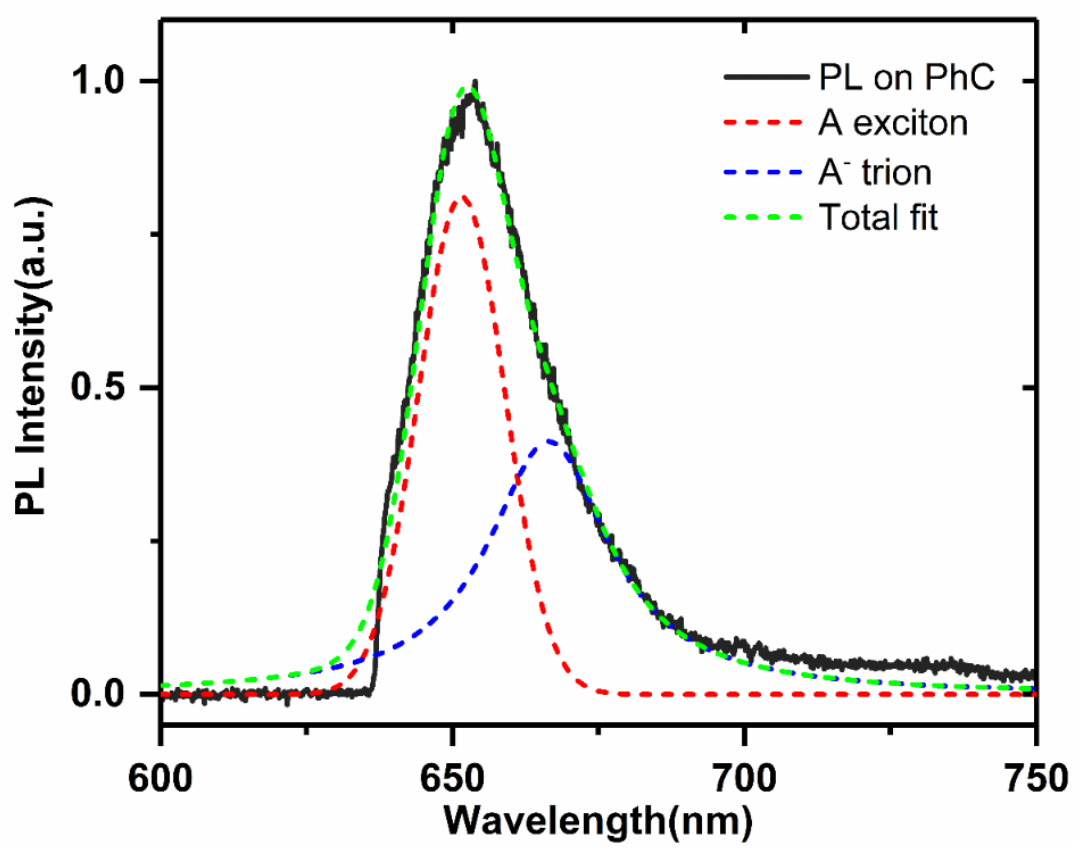

Fig. S4 Deconvolution of PL spectrum from $\mathrm{MoS}_{2}$ on the PhC. 


\section{S6. Angle-resolved transmittance along $\Gamma-M$ direction}

We also perform the angle-resolved transmission measurement along the $\Gamma$ $\mathrm{M}$ direction with p-polarized incident light in Fig. S6. The resonance near $650 \mathrm{~nm}$, which agrees with the PL peak position from aperture 3 in Fig. S5b, overlaps with the overall PL spectrum at $18^{\circ}$, resulting in directional enhancement at the same angle. The group velocity near this resonance, which can be characterized by the slope in the band diagram, is larger than the one at the $\mathrm{X}$ point in the main text. Also, the spectral overlap only covers the "tip" of this photonic band. Both reasons cause a narrower angular spread in the far-field radiation pattern compared with the one at the $\mathrm{X}$ point in Fig. 2e of the main text.

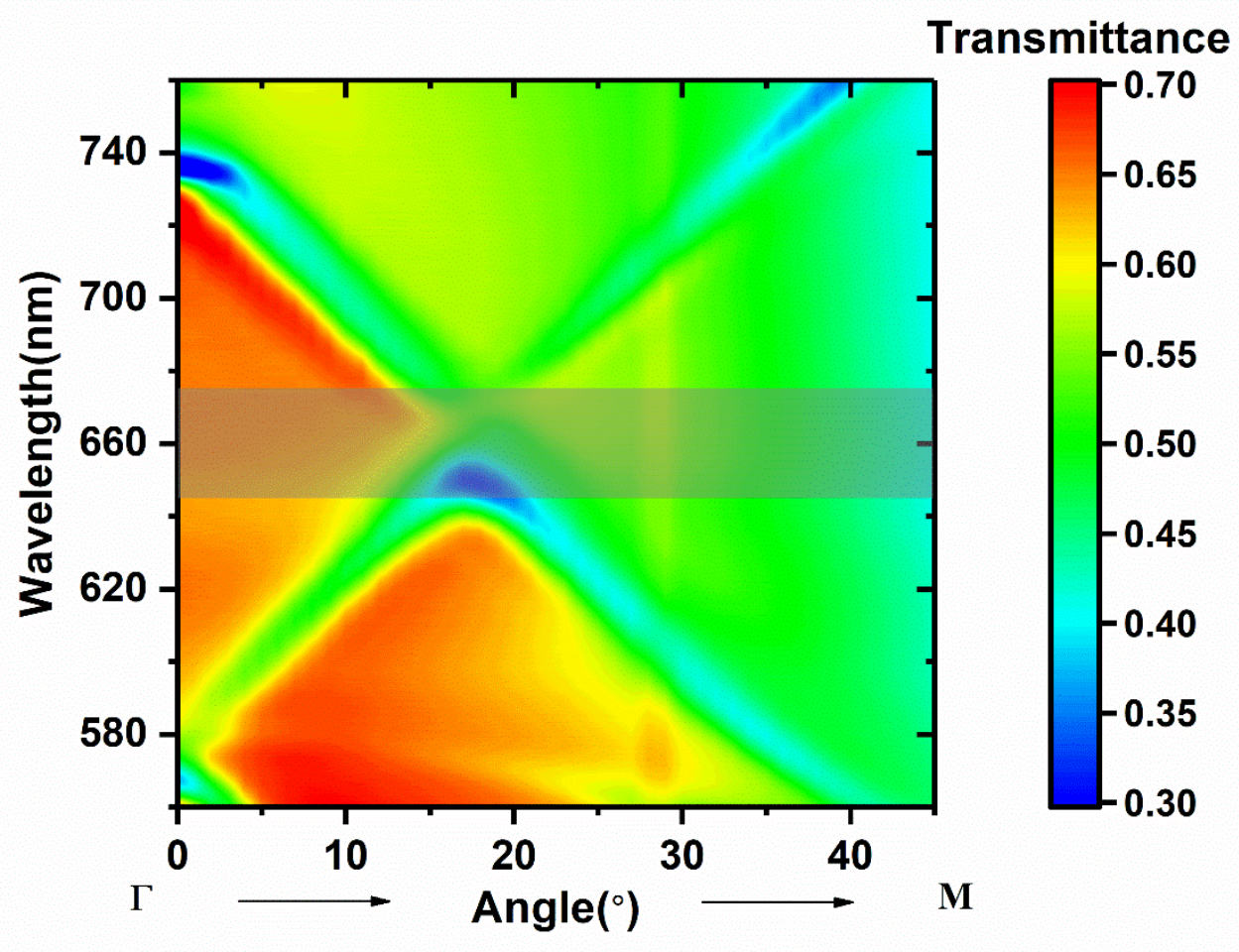

Fig. S6 The angle-resolved transmittance measured along $\Gamma-\mathrm{M}$ direction with p-polarized light. 


\section{S7. Absence of valley coherence}

To verify the potential existence of valley coherence of monolayer $\mathrm{MoS}_{2}$ in our device, we measured co- and cross-polarization PL with respect to the pump in the back focal plane. $\{\alpha, \beta\}$ means pump laser is at $\alpha$-polarization and PL is collected along $\beta$-direction. If valley coherence exists, pump laser at $\mathrm{x}$-polarization will create more x-polarized excitons, which will generate stronger PL in $\mathrm{x}$-direction because they are coupled into the p-polarized resonance of the PhC. However, Figs. S7a and S7b clearly show that PL intensity does not change when the pump laser polarization is rotated 90 degrees. We also calculate the valley polarization in each direction,

$$
\begin{aligned}
& \mathrm{p}_{\mathrm{x}}=\left(\mathrm{I}_{\mathrm{xx}}-\mathrm{I}_{\mathrm{yx}}\right) /\left(\mathrm{I}_{\mathrm{xx}}+\mathrm{I}_{\mathrm{yx}}\right)=0.016 \\
& \mathrm{p}_{\mathrm{y}}=\left(\mathrm{I}_{\mathrm{yy}}-\mathrm{I}_{\mathrm{xy}}\right) /\left(\mathrm{I}_{\mathrm{yy}}+\mathrm{I}_{\mathrm{xy}}\right)=-0.007
\end{aligned}
$$

which shows no increased valley coherence compared with monolayer $\mathrm{MoS}_{2}$ on a bare substrate $(\mathrm{p}=0.01 \pm 0.006)^{1}$.
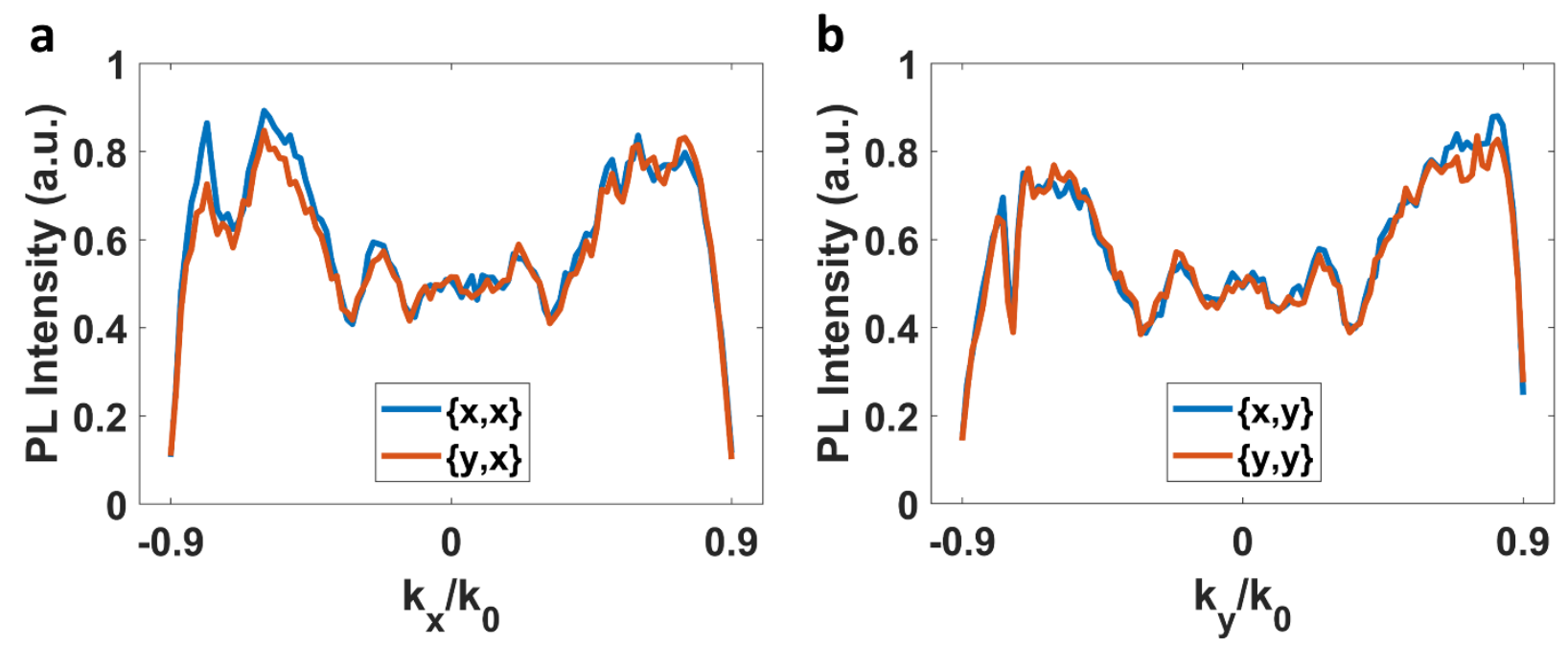

Fig. S6 (a) PL intensity along x-direction with different pump polarizations, (b) PL intensity along ydirection with different pump polarizations. 


\section{S8. $\mathrm{MoS}_{2}$ PL coupled with another TE-like band}

The $5^{\text {th }}$ TE-like band is highlighted in red solid line in Fig. S8a, which also crosses the PL spectral range. In the angle-resolved transmission spectra Fig. S8b, the resonance at the band-edge (purple circle) becomes invisible because the high radiative quality factor originated from the interference with the opposite band ${ }^{2}$ results in an infinitely small Fano feature in the transmission spectrum ${ }^{3}$. Also, the electric field profile in Fig. S8c shows no obvious enhancement in the hole area where the monolayer is suspended so the overall PL is still suppressed by the doping effect from the SiN substrate.

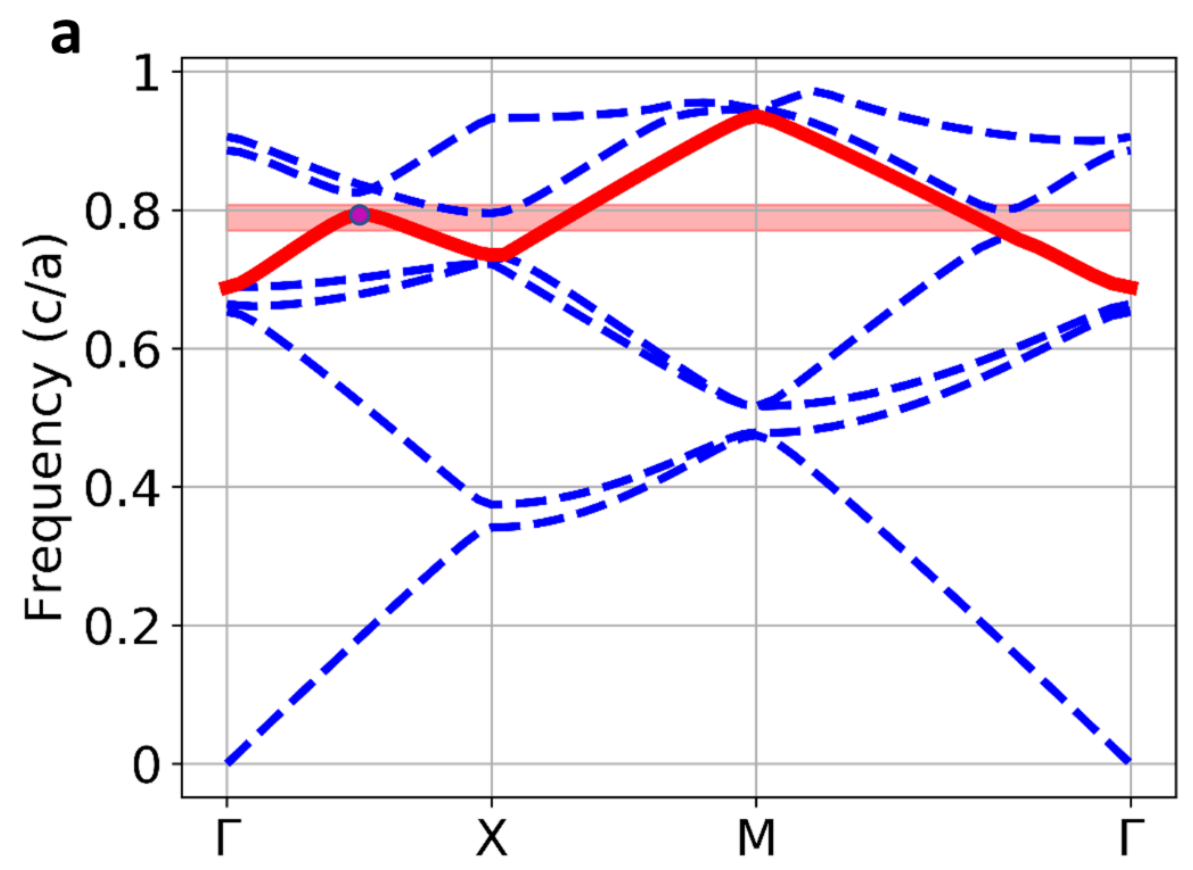




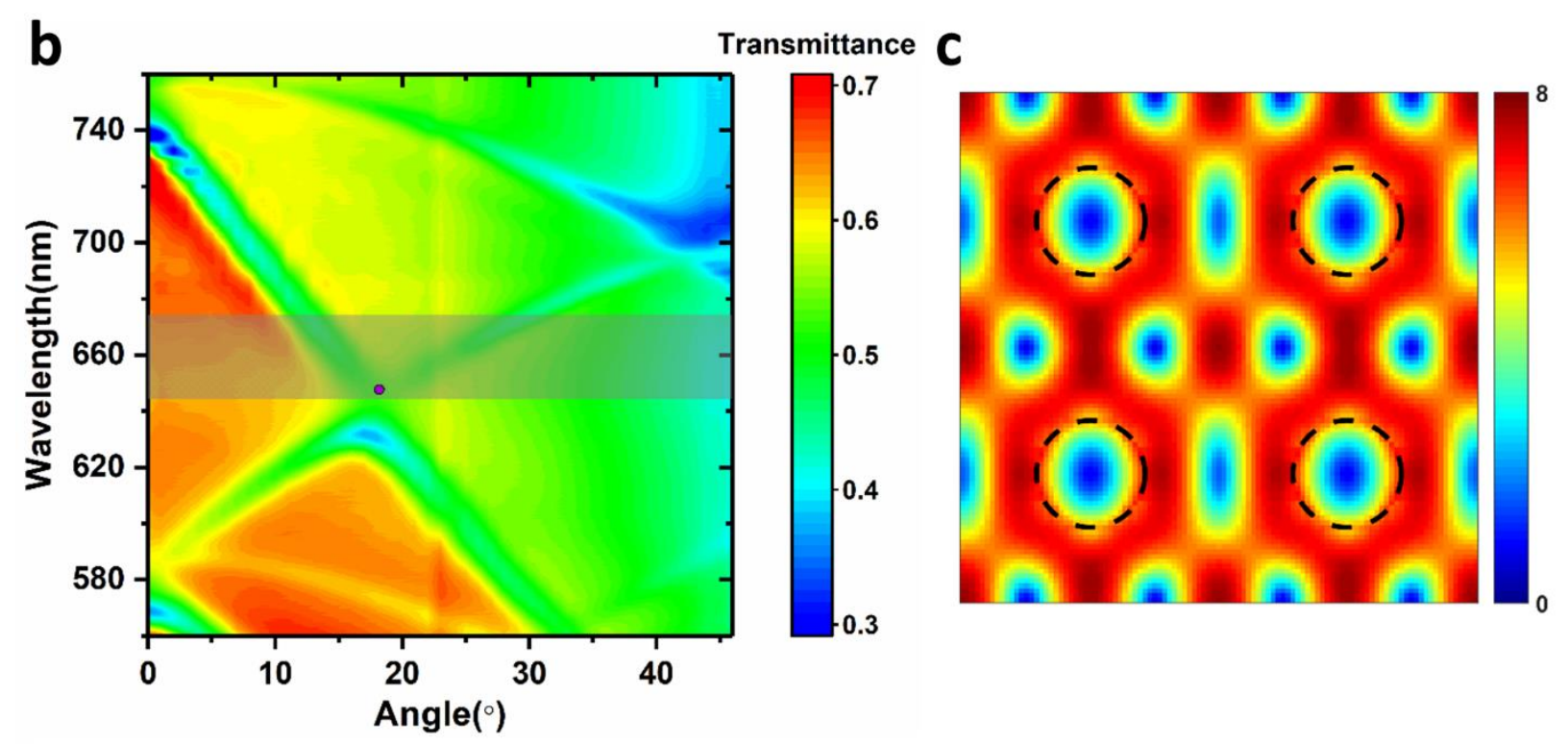

Fig. S8 (a) Band diagram for modes with even symmetry in $z$-direction. The red line represents the spolarized band that is across the PL spectral range (light red region). (b) The angle-resolved transmission spectra along $\Gamma$-X direction with s-polarized light. The purple circle indicates the resonance at the bandedge. (c) The E-field profile at the photonic band-edge. The field enhancement is mostly located on the SiN instead of the air holes.

\section{References}

1. Chen, Y.-J.; Cain, J. D.; Stanev, T. K.; Dravid, V. P.; Stern, N. P., Valley-polarized excitonpolaritons in a monolayer semiconductor. Nat. Photonics 2017, 11, 431-435.

2. Friedrich, H.; Wintgen, D., Interfering resonances and bound states in the continuum. Phys. Rev. A 1985, 32, 3231-3242.

3. Hsu, C. W.; Zhen, B.; Lee, J.; Chua, S. L.; Johnson, S. G.; Joannopoulos, J. D.; Soljacic, M., Observation of trapped light within the radiation continuum. Nature 2013, 499, 188-91. 